\title{
A Cooperative Mobile Satellite Communication System with the Dynamic Space-Time Coding
}

\author{
Gengrun Wang, ${ }^{1}$ Bangning Zhang, ${ }^{2}$ Kegang Pan, ${ }^{2}$ Aijun Liu, ${ }^{2}$ and Daoxing Guo ${ }^{2}$ \\ ${ }^{1}$ National Digital Switching System Engineering and Technological Research Center, Zhengzhou, Henan 450002, China \\ ${ }^{2}$ College of Communication Engineering, PLA University of Science and Technology, Nanjing, Jiangsu 210007, China \\ Correspondence should be addressed to Gengrun Wang; wanggengrun@gmail.com
}

Received 4 July 2014; Accepted 3 February 2015

Academic Editor: Swagatam Das

Copyright (c) 2015 Gengrun Wang et al. This is an open access article distributed under the Creative Commons Attribution License, which permits unrestricted use, distribution, and reproduction in any medium, provided the original work is properly cited.

\begin{abstract}
Channels of mobile satellite communications are affected by multipath fading and shadowing attenuation. At the same time, diversity gains are believed to improve the transmission reliability in fading channels. Considering that the traditional Space-Time Coding (STC) is not suitable for the cooperative mobile satellite communication, in this paper, a new cooperative mobile satellite communication system is proposed based on the Dynamic Space-Time Coding (D-STC). The transmitting energy of the proposed scheme is saved by avoiding forwarding erroneous signals in cooperative users. Meanwhile, this system benefits from diversity gains and the transmission is robust. Additionally, a closed-form expression of the outage probability for the proposed scheme is derived, and then it is demonstrated that this scheme is much better than the existing noncooperative scheme and schemes with traditional STCs in the outage performance. Finally, the analytical result is supported and validated by numerical simulations.
\end{abstract}

\section{Introduction}

As communication demand increases, it is required that communications should provide reliable and high speed service to users, regardless of where or when users need it. Although terrestrial cellular mobile networks can provide communication service to mobile users, they are limited by the coverage of cellular, cost, and so on.

The mobile satellite communication has attracted much interest and is considered as a useful way to provide communication service [1]. Besides wide coverage, large communication capacity, long distance communication support, and so forth, the satellite communication has the advantage of support of mobile users. Moreover, this communication strategy is not affected by the topography, and its cost does not increase with the distance of end-users [2-4]. Considering advantages of the mobile satellite communication, which is a seamless connection and of low cost, it is suitable to take this communication strategy as an important part in future multimedia communications.

A next-generation network (NGN) is a future packetbased network being developed by International Telecommunication Union (ITU). In particular, terrestrial wireless communications and satellite-based networks could all contribute to the NGN in terms of hybrid/integrated networks, providing ubiquitous and universal broadband IP-based services to mobile users. Evans et al. examined the role of the satellite communication in future telecommunication networks and service provision. They outlined some future ways forward for satellites and discussed research challenges and technology advances needed to facilitate this integrated approach [5].

Considering the specificity of mobile satellite communication channels, it is believed that the line-of-sight (LOS) communication is necessary for the mobile satellite communication, due to the huge path-loss and the effect of shadowing. Additionally, because of the mobility of land users, channels of mobile land users also suffer from the multipath effect. Thus, spatial diversity is useful to provide reliable communication service. Paillassa et al. proposed cooperative transmission schemes for the delivery of satellite services; these schemes provide spatial diversity just like MIMO transmission schemes and the coverage area is extended [6]. Nasser and Helard proposed a Layered SpaceTime Block Code (LSTBC) for MIMO schemes in hybrid satellite-terrestrial transmission of NGH systems; the code in 
these schemes is based on a layered construction designed to be efficient in shadowing regions [7]. Kota et al. focused on the design of integrated/hybrid systems taking into account physical, MAC, and network layers issues; they also described system examples and standards and then discussed cooperative diversity techniques and traffic engineering issues for overflow traffic [8].

In this paper, we propose a cooperative mobile satellite communication system, which is suitable for fading mobile satellite channels. By adopting the Space-Time Coding (STC) dynamically, this scheme can save the transmitting energy by avoiding forwarding erroneous signals of cooperative users. At the same time, every user will benefit from the diversity gain. Moreover, we also derive the closed-form expression of the outage probability for the proposed scheme. Given the theory analysis and simulations, it is proved that our scheme can effectively decrease the outage probability of the communication. Thus, the transmission reliability is improved and the system is more robust. Additionally, compared with existing cooperative schemes such as the scheme with full-STC [9] and the scheme with opportunisticSTC [10], the transmitting energy in the cooperative phase of the proposed scheme is more efficient, and then the outage performance is more attractive.

The remainder of the paper is organized as follows. In Section 2, we present the transmission procedure of the proposed scheme in detail. In Section 3, outage performance of the proposed scheme is analyzed. Simulation results are shown in Section 4. Finally, conclusions are drawn in Section 5 .

\section{System Model for the Cooperative Mobile Satellite Communication}

In this section, we describe the proposed cooperative mobile satellite communication system, which adopts the Dynamic Space-Time Coding (D-STC), in detail. We consider a mobile satellite communication system model with two cooperative land users $\left(U_{1}\right.$ and $\left.U_{2}\right)$ and one satellite $(S)$.

This model is depicted in Figure 1. We model the channel between the land user and the satellite $(U-S)$ as Rice fading with Rice factor $K$. Additionally, the channel between cooperative users $(U-U)$ is modeled as block Rayleigh fading. The fading coefficient of the channel $U_{j}-U_{i}$ is denoted by $h_{i j}$. It represents effects of path-loss, shadowing, and frequency nonselective fading and is modeled as a zeromean, independent, circularly symmetric complex Gaussian random variable with a variance of $\delta_{i j}^{2}$. The fading coefficients keep constant during one period and vary over different periods. Besides, transmissions suffer from additive white Gaussian noise (AWGN) and we model the noise at the input of node $i$ (i.e., $n_{i}$ ) as zero-mean, circularly symmetric, complex Gaussian random sequences with a variance of $N_{0}$. The noise $n_{i}$ at the input of different nodes is assumed to be independent and identically distributed (i.i.d.). The instantaneous and average received signal-to-noise ratio (SNR) is given, respectively, by $\gamma_{i j}:=\left|h_{i j}\right|^{2} \gamma$ and $\bar{\gamma}_{i j}:=$ $\delta_{i j}^{2} \gamma$, where $\gamma:=P_{x} / N_{0}$ denotes the SNR without channel

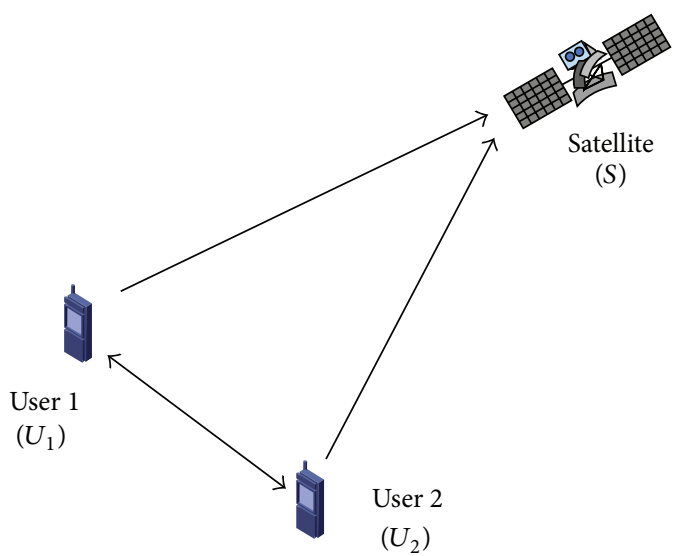

FIGURE 1: A mobile satellite communication system model with two cooperative users and one satellite.

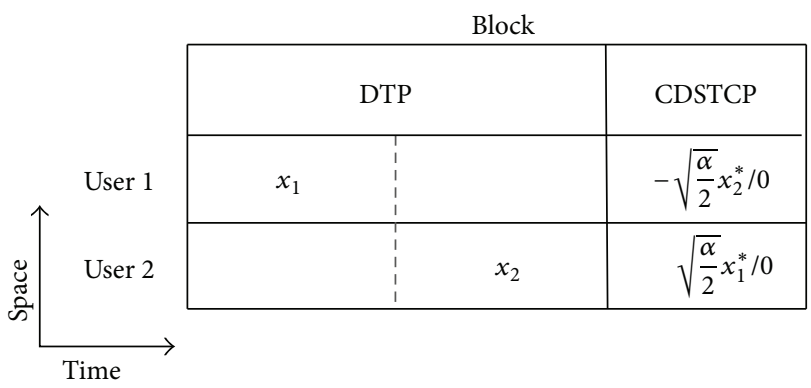

Figure 2: The time-division structure for the D-STC.

fading and $P_{x}$ is the average transmitting power. We assume that the channel state information is accurately measured at appropriate receivers via training symbols sent by transmit terminals, but not fully known by transmitters.

In this paper, we only consider the half-duplex mode of the cooperative land users, because current limitations in radio implementation preclude mobile land users from full-duplex operation. The transmission frame is divided into orthogonal subchannels, and the orthogonality is achieved by allocating nonoverlapping time slots to different users in this paper. The time-division channel allocation for this model is illustrated in Figure 2. In this figure, the transmission procedure is divided into two phases. The first two time slots compose the direct transmission phase (DTP), and the last time slot is the cooperative D-STC phase (CDSTCP). During the DTP, every user transmits its signal in the separated time slot and receives signals from its cooperative partner in another time slot. During the CDSTCP, users encode the information from their partners by the D-STC and then transmit the encoded signals simultaneously to the satellite. In the CDSTCP, $\alpha$ is the factor which controls the transmitting power and makes the average transmitting power in our scheme equal to that of the noncooperative scheme. In every block, the satellite receives separated signals from land users in the DTP and D-STC coded signals in the CDSTCP. 
For simplicity, we take $U_{1}$ as an example to describe the transmission procedure. In the first time slot of the DTP, $U_{1}$ transmits its signal to $U_{2}$ and $S$. Signals received by $U_{2}$ and $S$ are expressed as follows:

$$
\begin{aligned}
& y_{21}=h_{21} x_{1}+n_{21}, \\
& y_{1 s}=h_{1 s} x_{1}+n_{1 s},
\end{aligned}
$$

where $x_{1}$ represents the signal sent by $U_{1}$ and is drawn from a finite alphabet $\mathscr{A}_{x}, y_{i j}$ represents the signal of $U_{j}$ received by $U_{i}, y_{i s}$ represents the signal received by $S$ during the $i$ th time slot in the DTP, and $h_{i s}$ is the fading coefficient of channel $U_{i}$-S.

Then, $U_{2}$ attempts to retrieve information from the received signal by the maximum likelihood (ML) demodulation. The estimated signal is

$$
\widehat{x}_{1}=\arg \min _{x \in \mathscr{A}_{x}}\left\|y_{21}-h_{21} x\right\|^{2} .
$$

Next, $U_{2}$ will determine whether the information vector from $U_{1}$ is correctly decoded by using a cyclic redundancy check (CRC) [11]. If the estimated signal is correct (i.e., $\left.\hat{x}_{1}=x_{1}\right), U_{2}$ will encode the signal with the STC and transmit the coded signal in the CDSTCP. Otherwise, $U_{2}$ will keep silent in the CSTCP. As a result, energy will be saved by avoiding forwarding erroneous signals in the CDSTCP. Additionally, in our scheme, this saved energy is used to enhance the transmitting energy in the CDSTCP, when the decoded symbol is correct. In this case, the amplification factor of the transmitting energy is $\alpha$. It is a factor which makes the average transmitting power equal to that of the noncooperative scheme.

Meanwhile, $U_{1}$ works in the same way as described above. As a result, there are four cases at $S$ to decode received signals.

First, we consider case one when both $U_{1}$ and $U_{2}$ have correctly retrieved their partners' information. Then, in the CDSTCP, $S$ will obtain the signal encoded by the Alamouti Space-Time Coding [12]:

$$
y_{3 s}^{1}=\sqrt{\frac{\alpha}{2}}\left(-h_{1 s} x_{2}^{*}+h_{2 s} x_{2}^{*}\right)+n_{3 s} .
$$

Second, for case two and case three, when only one of the land users has correctly retrieved information of its partner, the signal received at $S$ is

$$
y_{3 s}^{2}=\sqrt{\frac{\alpha}{2}} h_{2 s} x_{1}^{*}+n_{3 s}
$$

or

$$
y_{3 s}^{3}=-\sqrt{\frac{\alpha}{2}} h_{1 s} x_{2}^{*}+n_{3 s} .
$$

Third, the case when neither $U_{1}$ nor $U_{2}$ has correctly retrieved information of its partner is denoted by case four. In this case, $S$ will not receive any information of land users in the CDSTCP.

According to the four cases above, there are four decoding types for $S$ to decode received signals.
For case one (full cooperation), $S$ will utilize the Alamouti space-time decoding and obtain

$$
\begin{aligned}
& {\left[\begin{array}{cc}
\sqrt{\frac{\alpha}{2}} h_{1 s}^{*} & h_{2 s} \\
\sqrt{\frac{\alpha}{2}} h_{2 s}^{*} & -h_{1 s}
\end{array}\right]\left[\begin{array}{c}
y_{1 s}+y_{2 s} \\
y_{3 s}^{1}
\end{array}\right]} \\
& =\sqrt{\frac{\alpha}{2}\left[\begin{array}{cc}
\left|h_{1 s}\right|^{2}+\left|h_{2 s}\right|^{2} & 0 \\
0 & \left|h_{1 s}\right|^{2}+\left|h_{2 s}\right|^{2}
\end{array}\right]\left[\begin{array}{l}
x_{1} \\
x_{2}
\end{array}\right]} \\
& +\left[\begin{array}{c}
\sqrt{\frac{\alpha}{2}} h_{1 s}^{*}\left(n_{1 s}+n_{2 s}\right)+h_{2 s} n_{3 s}^{*} \\
\sqrt{\frac{\alpha}{2}} h_{2 s}^{*}\left(n_{1 s}+n_{2 s}\right)-h_{1 s} n_{3 s}^{*}
\end{array}\right] .
\end{aligned}
$$

For case two and case three (half cooperation), the Alamouti space-time decoding is also utilized and results are as follows:

$$
\begin{aligned}
{\left[\begin{array}{ccc}
h_{1 s}^{*} & 0 & h_{2 s} \\
0 & h_{2 s}^{*} & 0
\end{array}\right]\left[\begin{array}{c}
y_{1 s} \\
y_{2 s} \\
y_{3 s}^{2 *}
\end{array}\right]=} & {\left[\begin{array}{cc}
\left|h_{1 s}\right|^{2}+\sqrt{\frac{\alpha}{2}}\left|h_{2 s}\right|^{2} & 0 \\
0 & \left|h_{2 s}\right|^{2}
\end{array}\right]\left[\begin{array}{c}
x_{1} \\
x_{2}
\end{array}\right] } \\
& +\left[\begin{array}{c}
h_{1 s}^{*} n_{1 s}+h_{2 s} n_{3 s}^{*} \\
h_{2 s}^{*} n_{2 s}
\end{array}\right]
\end{aligned}
$$

or

$$
\begin{aligned}
{\left[\begin{array}{ccc}
h_{1 s}^{*} & 0 & 0 \\
0 & h_{2 s}^{*} & -h_{1 s}
\end{array}\right]\left[\begin{array}{c}
y_{1 s} \\
y_{2 s} \\
y_{3 s}^{3^{*}}
\end{array}\right]=} & {\left[\begin{array}{cc}
\left|h_{1 s}\right|^{2} & 0 \\
0 & \sqrt{\frac{\alpha}{2}}\left|h_{1 s}\right|^{2}+\left|h_{2 s}\right|^{2}
\end{array}\right]\left[\begin{array}{l}
x_{1} \\
x_{2}
\end{array}\right] } \\
& +\left[\begin{array}{c}
h_{1 s}^{*} n_{1 s} \\
h_{2 s}^{*} n_{2 s}-h_{1 s} n_{3 s}^{*}
\end{array}\right] .
\end{aligned}
$$

For case four (no cooperation), the satellite will only utilize signals received in the DTP to obtain information of land users. Then, the decoding result is

$$
\left[\begin{array}{cc}
h_{1 s}^{*} & 0 \\
0 & h_{2 s}^{*}
\end{array}\right]\left[\begin{array}{c}
y_{1 s} \\
y_{2 s}
\end{array}\right]=\left[\begin{array}{cc}
\left|h_{1 s}\right|^{2} & 0 \\
0 & \left|h_{2 s}\right|^{2}
\end{array}\right]\left[\begin{array}{l}
x_{1} \\
x_{2}
\end{array}\right]+\left[\begin{array}{l}
h_{1 s}^{*} n_{1 s} \\
h_{2 s}^{*} n_{2 s}
\end{array}\right] .
$$

\section{Outage Performance}

In order to analyze the transmission performance, we will derive the outage probability expression of the proposed scheme. An outage occurs when the mutual information is lower than the information rate. This performance indicates the communication reliability over fading channels. Considering that two symbols are transmitted in three time slots over a block, the bit rate will be $3 / 2$ times that of the noncooperation scheme in fairness. 
At the beginning, we deduce occurrence probabilities of the four cases corresponding to different cooperation types, and then we will calculate outage probabilities under these cases.

When $U_{1}$ provides assistance to its partner (i.e., $U_{2}$ ), the mutual information of $U_{2}-U_{1}$ must be larger than the data rate of $U_{2}$ (i.e., $R_{2}$ ):

$$
I_{12}>R_{2} \text {. }
$$

Besides, the mutual information $I_{12}$ is calculated as

$$
I_{12}=\log _{2}\left(1+\left|h_{12}\right|^{2} \gamma\right) \text {. }
$$

By substituting (11) into (10), the occurrence probability of $U_{1}$ 's assistance to $U_{2}$ is calculated as

$$
\begin{aligned}
P_{12} & =P\left\{\log _{2}\left(1+\left|h_{12}\right|^{2} \gamma\right)>R_{2}\right\} \\
& =P\left\{\left|h_{12}\right|^{2}>\frac{2^{R_{2}}-1}{\gamma}\right\}=\exp \left(-\frac{2^{R_{2}}-1}{\gamma \sigma_{12}^{2}}\right) .
\end{aligned}
$$
as

$$
\alpha=\frac{1}{P_{12}} .
$$

In this way, the transmitting power of the proposed scheme in the CDSTCP is approximately equal to that of the noncooperative scheme for a fair comparison.

Then, the occurrence probability of the full cooperation (case one) is obtained as

$$
P_{f}=P_{21} \cdot P_{12},
$$

where $P_{21}$ is the occurrence probability of $U_{2}$ 's assistance to $U_{1}$.

Similarly, occurrence probabilities of half cooperation (case two and case three) and no cooperation (case four) will be derived as

$$
\begin{aligned}
& P_{h 1}=P_{21} \cdot\left(1-P_{12}\right), \\
& P_{h 2}=\left(1-P_{21}\right) \cdot P_{12}, \\
& P_{n}=\left(1-P_{21}\right) \cdot\left(1-P_{12}\right) .
\end{aligned}
$$

In the following, we will derive outage probabilities under the above-mentioned four cases.

First, under case one, the mutual information of $U_{1}-S$ is

$$
\begin{aligned}
I_{1 s}^{\text {full }} & =\log _{2}\left[1+\frac{(\alpha / 2)\left(\left|h_{1 S}\right|^{2}+\left|h_{2 S}\right|^{2}\right)^{2}}{\alpha\left|h_{1 S}\right|^{2}+\left|h_{2 S}\right|^{2}} \gamma\right] \\
& \geq \log _{2}\left(1+\frac{\left|h_{1 S}\right|^{2}+\left|h_{2 S}\right|^{2}}{2} \gamma\right) .
\end{aligned}
$$

Then, the outage probability under this case is

$$
\begin{aligned}
P_{\text {outage }}^{\text {full }} & \leq P\left[\log _{2}\left(1+\frac{\left|h_{1 S}\right|^{2}+\left|h_{2 S}\right|^{2}}{2} \gamma\right)<R_{1}\right] \\
& =P\left[\left|h_{1 S}\right|^{2}+\left|h_{2 S}\right|^{2}<\frac{2\left(2^{R_{1}}-1\right)}{\gamma}\right] \\
& =1-Q_{2}\left[\sqrt{2 K}, \sqrt{\frac{2\left(2^{R_{1}}-1\right)}{\gamma \sigma^{2}}}\right] .
\end{aligned}
$$

In this equation, $Q_{m}$ is the generalized Marcum $Q$ function [13], which is defined as

$$
Q_{m}(a, b)=Q_{1}(a, b)+e^{\left(a^{2}+b^{2}\right) / 2} \sum_{k=1}^{m-1}\left(\frac{b}{a}\right)^{k} I_{k}(a b),
$$

where $I_{k}(x)$ is the $k$-order modified Bessel function of the first kind:

$$
I_{k}(x)=\sum_{m=0}^{\infty} \frac{(x / 2)^{k+2 m}}{m ! \Gamma(k+m+1)} \quad(x \geq 0)
$$

and $Q_{1}(a, b)$ is

$$
Q_{1}(a, b)=e^{-\left(a^{2}+b^{2}\right) / 2} \sum_{k=1}^{m-1}\left(\frac{a}{b}\right)^{k} I_{k}(a b) .
$$

Second, the mutual information of $U_{1}-S$ under case two is calculated as

$$
\begin{aligned}
I_{1 s}^{h 1} & =\log _{2}\left[1+\frac{\left(\left|h_{1 S}\right|^{2}+\sqrt{\alpha / 2}\left|h_{2 S}\right|^{2}\right)^{2}}{\left|h_{1 S}\right|^{2}+\left|h_{2 S}\right|^{2}} \gamma\right] \\
& \geq \log _{2}\left(1+\frac{\alpha\left(\left|h_{1 S}\right|^{2}+\left|h_{2 S}\right|^{2}\right)}{2} \gamma\right) .
\end{aligned}
$$

Then, the outage probability under this case is

$$
\begin{aligned}
P_{\text {outage }}^{h 1} & \leq P\left[\log _{2}\left(1+\frac{\alpha\left(\left|h_{1 S}\right|^{2}+\left|h_{2 S}\right|^{2}\right)}{2} \gamma\right)<R_{1}\right] \\
& =P\left[\left|h_{1 S}\right|^{2}+\left|h_{2 S}\right|^{2}<\frac{2\left(2^{R_{1}}-1\right)}{\alpha \gamma}\right] \\
& =1-Q_{2}\left[\sqrt{2 K}, \sqrt{\frac{2\left(2^{R_{1}}-1\right)}{\alpha \gamma \sigma^{2}}}\right] .
\end{aligned}
$$

Third, the outage probability of the link $U_{1}-S$ under case three or case four is calculated as

$$
\begin{aligned}
P_{\text {outage }}^{h 2 / \text { no }} & =P\left[\log _{2}\left(1+\left|h_{1 S}\right|^{2} \gamma\right)<R_{1}\right] \\
& =P\left(\left|h_{1 S}\right|^{2}<\frac{2^{R_{1}}-1}{\gamma}\right) \\
& =1-Q_{1}\left(\sqrt{2 K}, \sqrt{\frac{2^{R_{1}}-1}{\gamma \sigma^{2}}}\right) .
\end{aligned}
$$


As a result, the outage probability of the link $U_{1}-S$ in the proposed scheme is obtained by

$$
P_{\text {outage }}=P_{f} \cdot P_{\text {outage }}^{\text {full }}+P_{h 1} \cdot P_{\text {outage }}^{h 1}+\left(P_{h 2}+P_{n}\right) \cdot P_{\text {outage }}^{h 2 / \text { no }}
$$

By substituting (14)-(15) and (17)-(23) into (24), this outage probability is rewritten as

$$
\begin{aligned}
P_{\text {outage }} \leq & \exp \left(-\frac{2^{R_{1}}-1}{\gamma \sigma_{12}^{2}}\right) \cdot \exp \left(-\frac{2^{R_{2}}-1}{\gamma \sigma_{21}^{2}}\right) \\
& \cdot\left\{1-Q_{2}\left[\sqrt{4 K}, \sqrt{\left.\left.\frac{2\left(2^{R_{1}}-1\right)}{\gamma \sigma^{2}}\right]\right\}}\right.\right. \\
& +\exp \left(-\frac{2^{R_{1}}-1}{\gamma \sigma_{12}^{2}}\right) \cdot\left[1-\exp \left(-\frac{2^{R_{2}}-1}{\gamma \sigma_{21}^{2}}\right)\right] \\
& \cdot\left\{1-Q_{2}\left[\sqrt{4 K}, \sqrt{\left.\left.\frac{2\left(2^{R_{1}}-1\right)}{\alpha \gamma \sigma^{2}}\right]\right\}}\right.\right.
\end{aligned}
$$$$
\begin{aligned}
{\left[\begin{array}{cc}
\sqrt{\frac{1}{2}} h_{1 s}^{*} & h_{2 s} \\
\sqrt{\frac{1}{2}} h_{2 s}^{*}-h_{1 s}
\end{array}\right]\left[\begin{array}{c}
y_{1 s}+y_{2 s} \\
y_{3 s}^{*}
\end{array}\right]=} & \sqrt{\frac{1}{2}}\left[\begin{array}{cc}
\left|h_{1 s}\right|^{2}+\left|h_{2 s}\right|^{2} & 0 \\
0 & \left|h_{1 s}\right|^{2}+\left|h_{2 s}\right|^{2}
\end{array}\right]\left[\begin{array}{c}
x_{1} \\
x_{2}
\end{array}\right] \\
+ & {\left[\begin{array}{c}
\sqrt{\frac{1}{2}} h_{1 s}^{*}\left(n_{1 s}+n_{2 s}\right)+h_{2 s} n_{3 s}^{*}+\sqrt{\frac{1}{2}}\left|h_{2 s}\right|^{2}\left(\hat{x}_{1}-x_{1}\right)-\sqrt{\frac{1}{2}} h_{1 s}^{*} h_{2 s}\left(\hat{x}_{2}-x_{2}\right) \\
\sqrt{\frac{1}{2}} h_{2 s}^{*}\left(n_{1 s}+n_{2 s}\right)-h_{1 s} n_{3 s}^{*}+\sqrt{\frac{1}{2}}\left|h_{1 s}\right|^{2}\left(\hat{x}_{2}-x_{2}\right)-\sqrt{\frac{1}{2}} h_{2 s}^{*} h_{1 s}\left(\hat{x}_{1}-x_{1}\right)
\end{array}\right] . }
\end{aligned}
$$

$$
\begin{aligned}
& +\left[1-\exp \left(-\frac{2^{R_{1}}-1}{\gamma \sigma_{12}^{2}}\right)\right] \\
& \cdot\left\{1-Q_{1}\left(\sqrt{2 K}, \sqrt{\frac{2^{R_{1}}-1}{\gamma \sigma^{2}}}\right)\right\} .
\end{aligned}
$$

At the same time, we can derive the outage probability for the scheme with full-STC, which was proposed by Scutari and Barbarossa [9]. In this traditional scheme, the distributed Alamouti Space-Time Coding was adopted to achieve the cooperative diversity, no matter whether users had decoded their cooperative partners' information in the cooperative phase successfully or not. To be fair, in the following derivation, the amplification factor of the transmitting energy is $1 / 2$ in the cooperative phase. As a result, signals received by the satellite during the whole block are

$$
\left[\begin{array}{c}
y_{1 s} \\
y_{2 s} \\
y_{3 s}
\end{array}\right]=\left[\begin{array}{c}
h_{1 s} x_{1} \\
h_{2 s} x_{2} \\
\frac{\left(-h_{1 s} \widehat{x}_{2}^{*}+h_{2 s} \widehat{x}_{1}^{*}\right)}{\sqrt{2}}
\end{array}\right]+\left[\begin{array}{c}
n_{1 s} \\
n_{2 s} \\
n_{3 s}
\end{array}\right] .
$$

By adopting the Alamouti decoding, the satellite will decode signals of land users as
And then, the outage probability of the scheme with fullSTC is calculated as

$$
\begin{aligned}
P_{\text {outage }}^{\text {full-STC }} & \\
= & P\left\{\log _{2}\left(1+\left|h_{21}\right|^{2} \gamma\right)>R_{2}\right\} \\
& \cdot P\left\{\log _{2}\left(1+\left|h_{12}\right|^{2} \gamma\right)>R_{1}\right\} \\
& \cdot P\left[\log _{2}\left(1+\frac{\left|h_{1 S}\right|^{2}+\left|h_{2 S}\right|^{2}}{2} \gamma\right)<R_{1}\right] \\
& +P\left\{\log _{2}\left(1+\left|h_{21}\right|^{2} \gamma\right)<R_{2}\right\} \\
& \cdot P\left\{\log _{2}\left(1+\left|h_{12}\right|^{2} \gamma\right)>R_{1}\right\}
\end{aligned}
$$




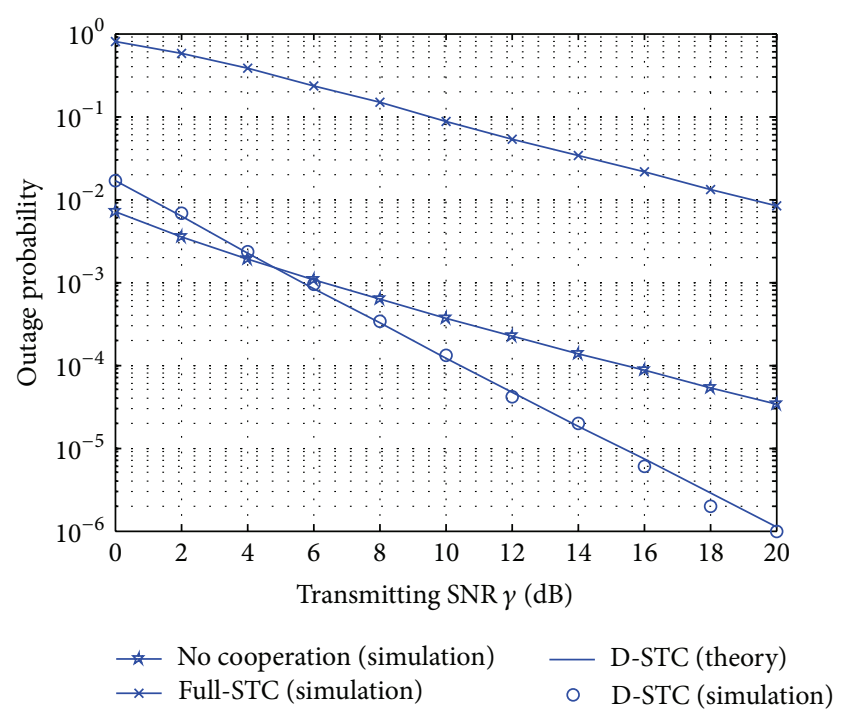

FIGURE 3: Outage probabilities of existing schemes and the proposed D-STC scheme.

$$
\begin{aligned}
& \cdot P\left\{\log _{2}\left(1+\left|h_{12}\right|^{2} \gamma\right)<R_{1}\right\} \\
& \cdot P\left\{\log _{2}[1\right. \\
& \left.\quad+\frac{\left(\left|h_{1 S}\right|^{2}+\left|h_{2 S}\right|^{2}\right)^{2}}{2\left(\left|h_{1 S}\right|^{2}+\left|h_{2 S}\right|^{2}+\left|h_{2 S}\right|^{4} \gamma+\left|h_{1 S}\right|^{2}\left|h_{2 S}\right|^{2} \gamma\right)} \gamma\right] \\
& \left.\quad<R_{1}\right\} .
\end{aligned}
$$

Although it is challenging to derive the exact closedform expression for this outage probability of the scheme with traditional full-STC, in the next section, we will give the result of simulations and compare it with that of the proposed scheme and the traditional noncooperative scheme.

\section{Simulation}

In this section, we will verify the analysis of the outage probability for practical SNR values. We present outage probabilities of the link from one land user to the satellite in different settings. The transmitting SNR $\gamma$ denotes the SNR without channel fading. The channel between the land user and the satellite is Rice fading with a Rice factor $K$, and the channel between land users is Rayleigh fading with a variance $\delta_{u}^{2}$. Unless specified otherwise, the Rice factor is set as $K=7$ and the Rayleigh variance is set as $\delta_{u}^{2}=1$. Additionally, the data rate for traditional noncooperative scheme is set as $R=1$ for comparison.

Figure 3 shows outage probabilities of the proposed scheme together with those of the scheme with full-STC and

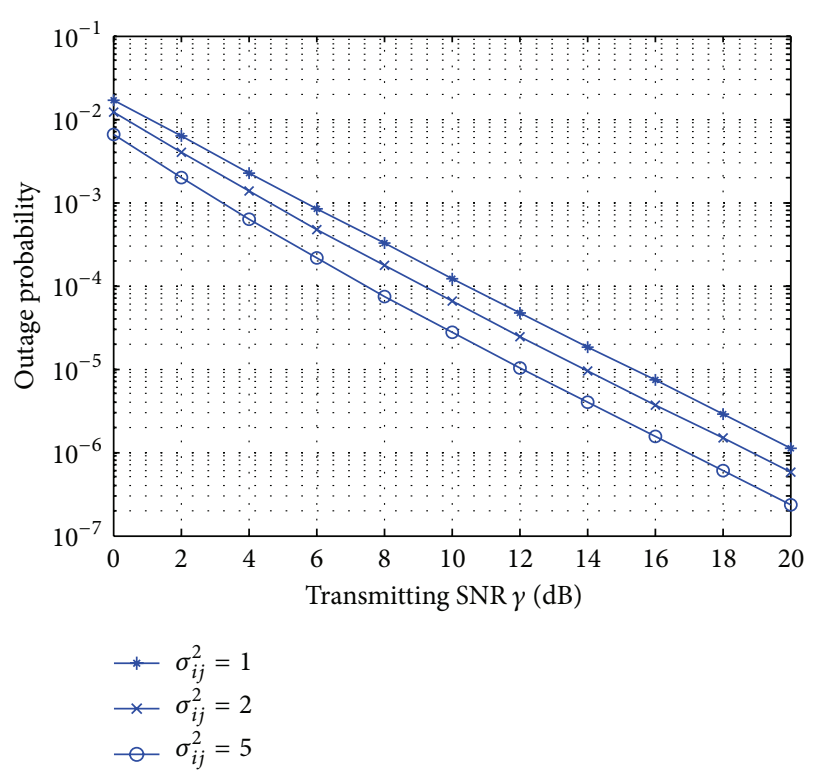

FIGURE 4: Outage probabilities of the proposed scheme in various $U-U$ channel properties.

the traditional scheme with no cooperation. In this figure, the outage probability of the proposed scheme is effectively decreased. In addition, it is noticed that the scheme with full-STC is even worse than the scheme without cooperation. That is because of the LOS path, which exists in the channel between the user and the satellite. This path is much stronger than the multipath, and then the channel property between land users is worse than the channel property between the land user and the satellite. This leads to the fact that cooperative users forward the erroneous signal with high probability. Given this, the proposed scheme can effectively avoid the error propagation. Additionally, as we can see, when the transmitting SNR is larger than $5 \mathrm{~dB}$, outage performance of the proposed scheme is better than that of the noncooperative scheme. The decrease of the outage probability indicates that our scheme achieves diversity order of two. Then, the proposed scheme benefits from cooperation. In addition, analysis of the proposed scheme in theory accords well with practical simulations. Thus, the closed-form expression of the outage probability for the proposed scheme is a useful tool to analyze the performance of this scheme.

The outage performance of the proposed scheme in various $U-U$ channel property settings is demonstrated in Figure 4 . With the improvement of the channel property between cooperative users, the probability of users correctly obtaining signals from each other is increased. As a result, users can provide the benefit of diversity gains to their partners to improve the outage performance. Then, it is suitable that every user chooses neighbor one as its cooperative partner, due to the better channel property. On the other hand, channel properties between cooperative users do not affect the diversity order, and then all corresponding scenarios achieve the diversity order of two. In order to obtain more diversity gains, it is better to design more flexible 


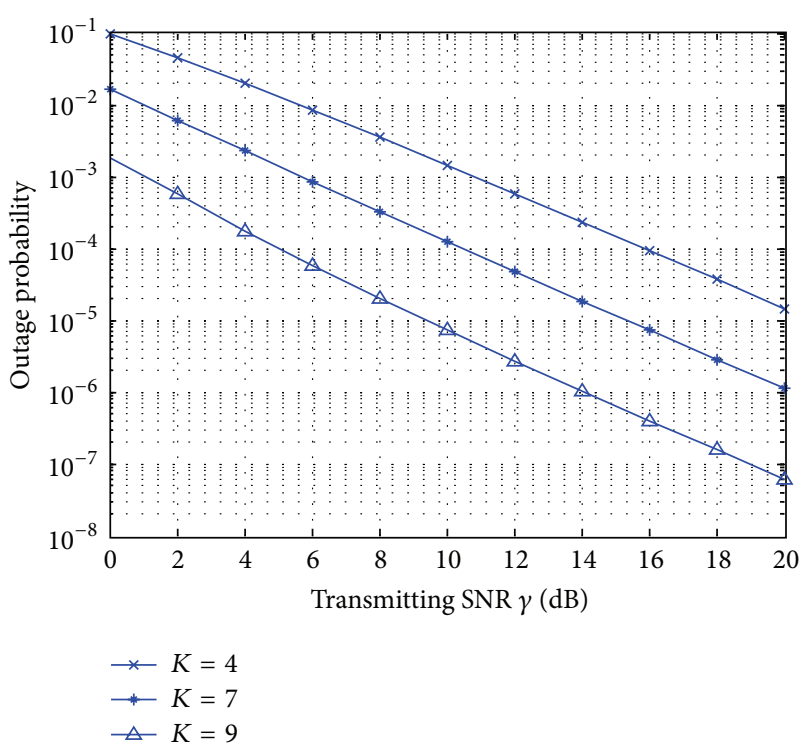

FIGURE 5: Outage probabilities of the proposed scheme in different $U-S$ channel properties.

schemes, which support more cooperative users to increase the diversity order.

Figure 5 shows simulation results of outage probabilities in different $U-S$ channel property settings. With the increase of elevation angles, the Rice factor $K$ of the $U-S$ channel is approximately changing from 2 to 12 [14]. In Figure 5, it is clear that better $U-S$ channel property can effectively decrease the outage probability. With the increase of the Rice factor $K$, the signal of the LOS path is much stronger than the multipath. As a result, the LOS path dominates the performance of the mobile satellite communication system. At the same time, with the benefit of cooperative diversity gains, the outage performance of the proposed scheme can be improved even more.

Figure 6 illustrates outage probabilities for different multiplexing gains. In this simulation, the data rate $R$ is defined as a function of multiplexing gain $r(R=r \cdot \log (1+$ $\gamma)$ ). This figure shows the relationship between the diversity gain and the multiplexing gain. As we can see, the outage probability is increased and the diversity order is decreased when multiplexing gains are increased from 0.2 to 0.8 . Given this, there is a tradeoff between the diversity gain and the multiplexing gain. Thus, we should make a choice between the transmission reliability and the efficiency for different communication circumstances. In order to utilize the diversity gain to improve outage performance, we should decrease the multiplexing gain at the cost of transmission efficiency. In other circumstances, the scheme can work in reverse.

\section{Conclusion}

In this paper, we have proposed a cooperative mobile satellite communication system with the D-STC. This scheme utilizes diversity gains to improve the outage performance of communications. By forwarding signals of cooperative partners

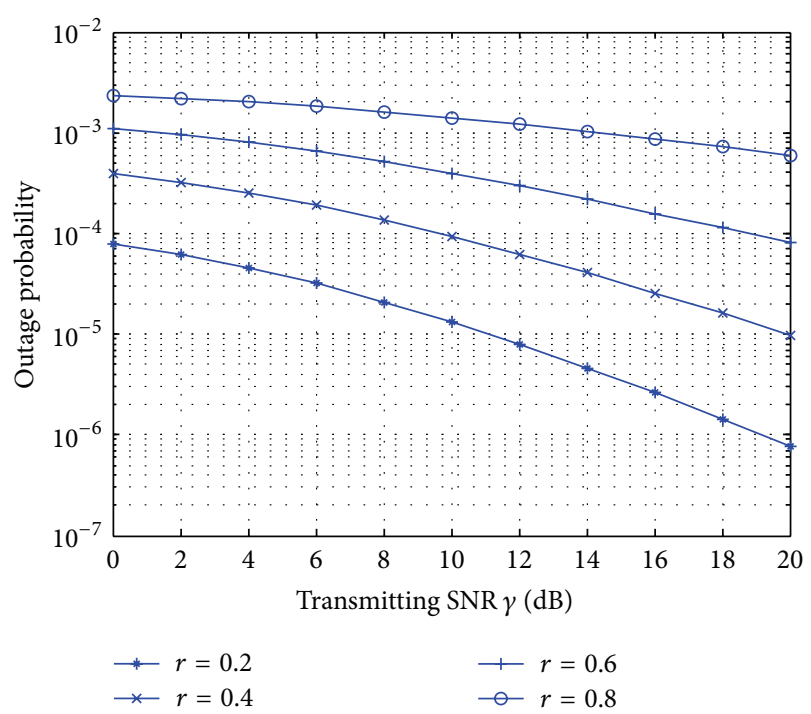

FIGURE 6: Outage probabilities of the proposed scheme with different multiplexing gains.

dynamically, the utilization of the transmitting energy is more efficient. Moreover, we have derived the closed-form expression of the outage probability for the proposed scheme. Through the analysis, the outage probability is effectively decreased. And then, the transmitting energy is saved. Simulation results demonstrate that, by selecting the neighbor user as the cooperative one, our scheme can achieve desirable outage performance. Additionally, there is a tradeoff between the diversity gain and multiplexing gain. Given this, we can design different scenarios for different resource limited communication circumstances.

In future research, the implementation of the proposed scheme and the design of the scheme, which supports more cooperative users to obtain more diversity gains, are two aspects to be considered.

\section{Conflict of Interests}

The authors declare that they have no conflict of interests.

\section{Acknowledgment}

The authors would like to thank Lu Xia for his helpful suggestions in the revision of this paper.

\section{References}

[1] T. T. Han, Digital Satellite Communication, Tata McGraw-Hill Education, 1990.

[2] A. Gotta and P. Barsocchi, "Experimental video broadcasting in DVB-RCS/S2 with land mobile satellite channel: a reliability issue," in Proceedings of the IEEE International Workshop on Satellite and Space Communications (IWSSC '08), pp. 234-238, 2008.

[3] ETSI TR 102 662, Satellite Earth Stations and Systems (SES): Advanced Satellite Based Scenarios and Architectures for Beyond $3 G$ Systems, 2010. 
[4] TTAR, "Requirement and system architecture for the satellite component of IMT-advanced," Tech. Rep. TTAR-06.0067, 2010.

[5] B. Evans, M. Werner, E. Lutz et al., "Integration of satellite and terrestrial systems in future multimedia communications," IEEE Wireless Communications, vol. 12, no. 5, pp. 72-80, 2005.

[6] B. Paillassa, B. Escrig, R. Dhaou, M.-L. Boucheret, and C. Bes, "Improving satellite services with cooperative communications," International Journal of Satellite Communications and Networking, vol. 29, no. 6, pp. 479-500, 2011.

[7] Y. Nasser and J.-F. Helard, "Layered space-time block code for hybrid satellite-terrestrial broadcasting systems," International Journal of Satellite Communications and Networking, vol. 30, no. 3, pp. 113-129, 2012.

[8] S. Kota, G. Giambene, and S. Kim, "Satellite component of NGN: integrated and hybrid networks," International Journal of Satellite Communications and Networking, vol. 29, no. 3, pp. 191208, 2011.

[9] G. Scutari and S. Barbarossa, "Distributed space-time coding for regenerative relay networks," IEEE Transactions on Wireless Communications, vol. 4, no. 5, pp. 2387-2399, 2005.

[10] Y. Zou, Y.-D. Yao, and B. Zheng, "Opportunistic distributed space-time coding for decode-and-forward cooperation systems," IEEE Transactions on Signal Processing, vol. 60, no. 4, pp. 1766-1781, 2012.

[11] L. Xiao, T. E. Fuja, J. Kliewer, and J. Costello, "A network coding approach to cooperative diversity," IEEE Transactions on Information Theory, vol. 53, no. 10, pp. 3714-3722, 2007.

[12] S. M. Alamouti, "A simple transmit diversity technique for wireless communications," IEEE Journal on Selected Areas in Communications, vol. 16, no. 8, pp. 1451-1458, 1998.

[13] B. Sklar, Digital Communications. Volume 2, Prentice Hall, Upper Saddle River, NJ, USA, 2001.

[14] G. E. Corazza and F. Vatalaro, "A statistical model for land mobile satellite channels and its application to nongeostationary orbit systems," IEEE Transactions on Vehicular Technology, vol. 43, no. 3, pp. 738-742, 1994. 


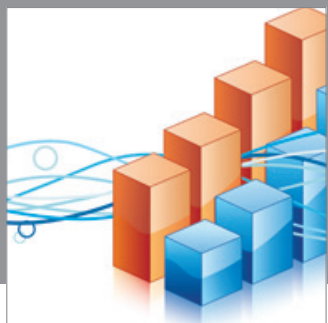

Advances in

Operations Research

mansans

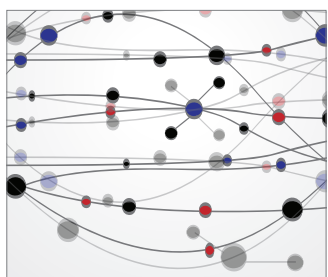

The Scientific World Journal
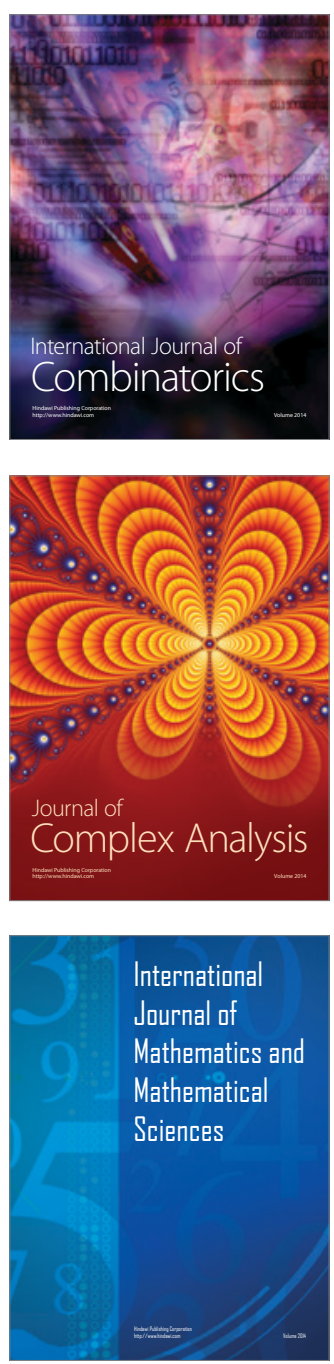
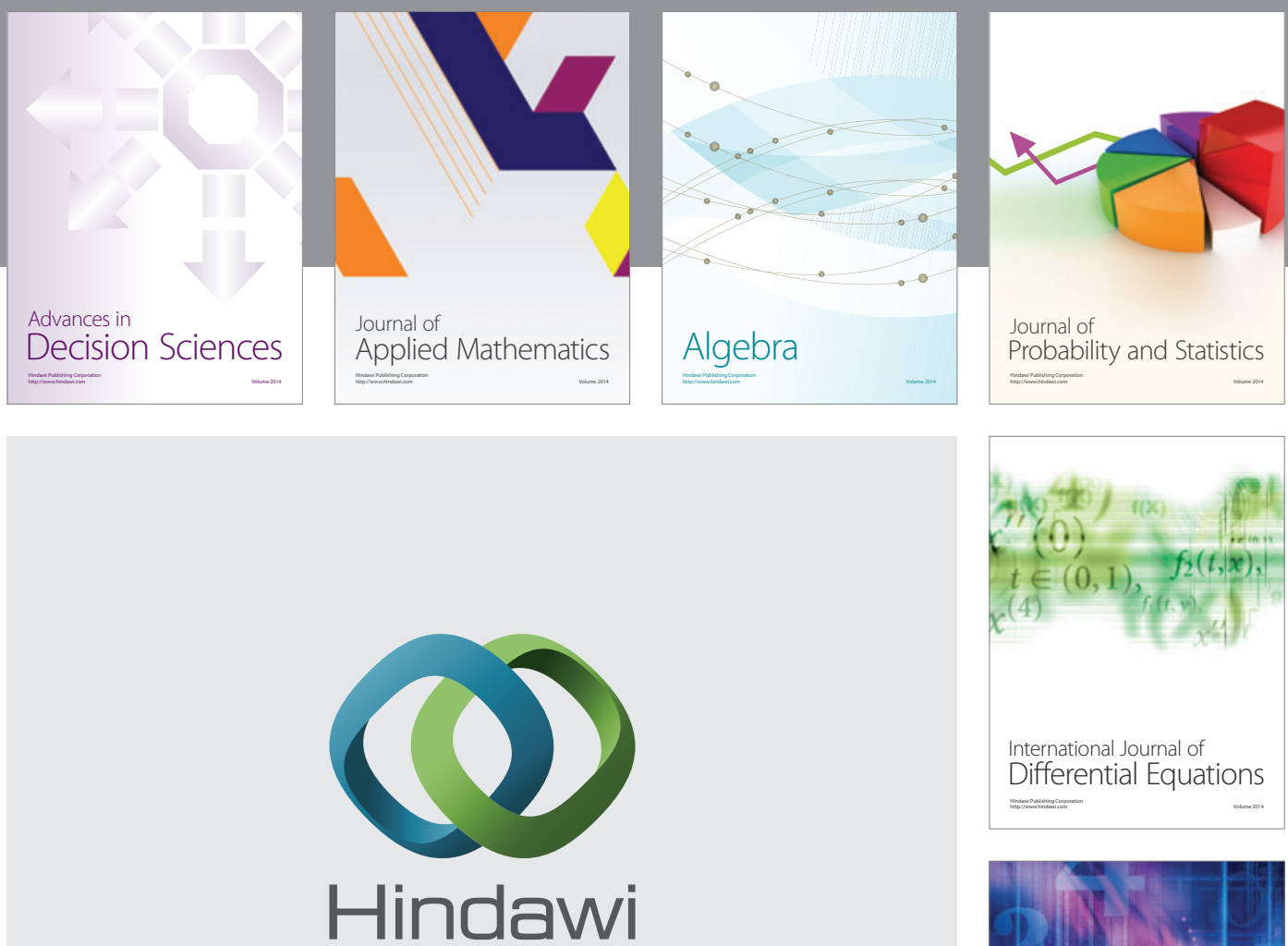

Submit your manuscripts at http://www.hindawi.com
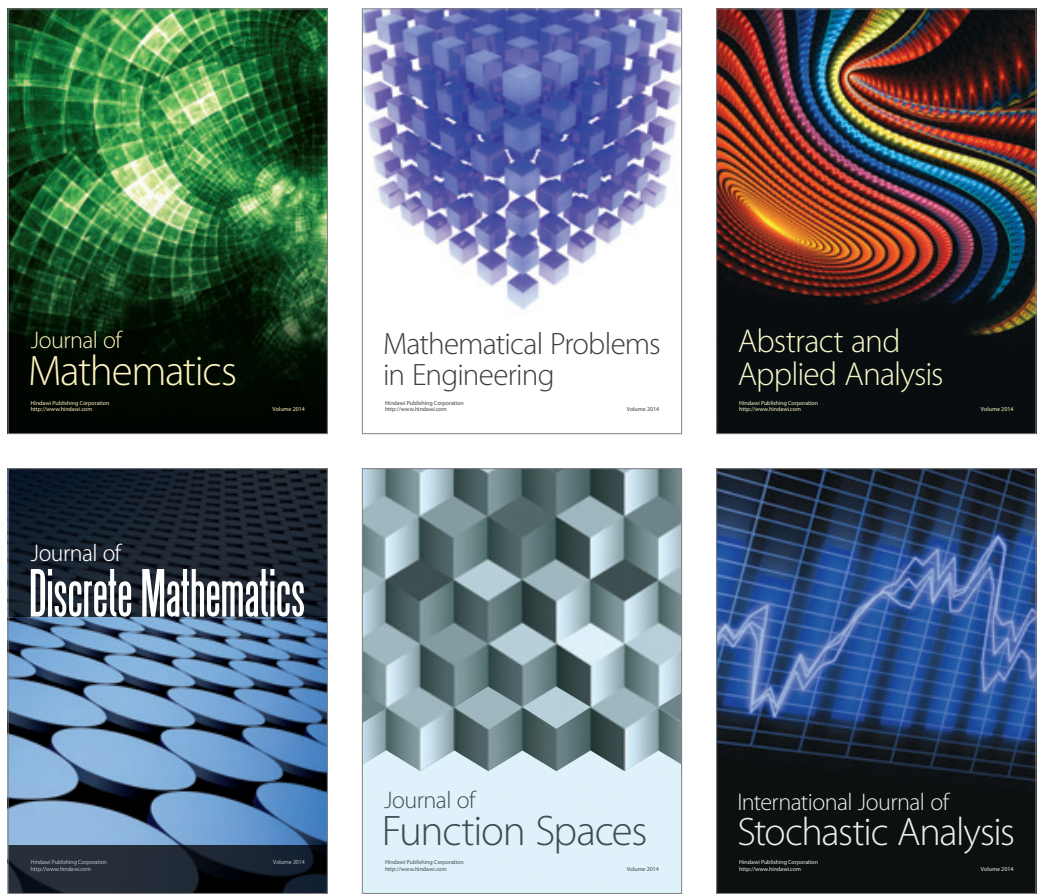

Journal of

Function Spaces

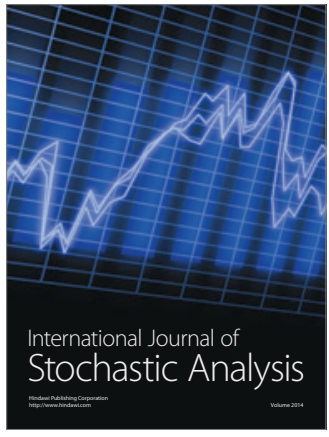

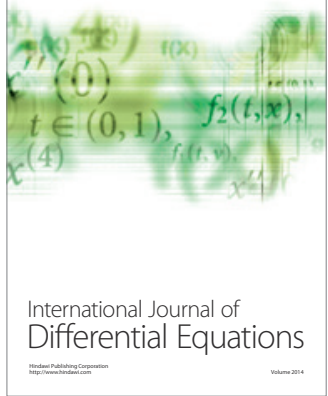
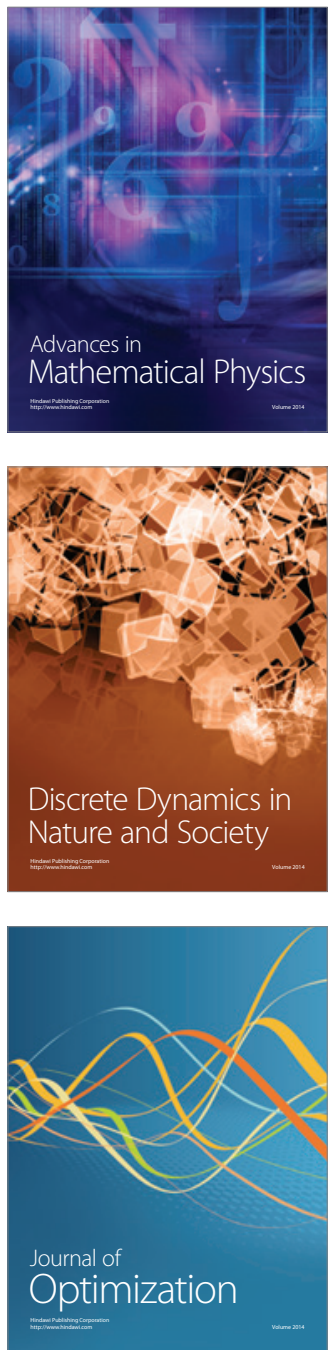